\title{
Asymptotic problems for fourth-order nonlinear differential equations
}

\author{
Miroslav Bartušek and Zuzana Došlá*
}

Dedicated to Jean Mawhin on occasion of his seventieth birthday.

"Correspondence:

dosla@math.muni.cz

Faculty of Science, Masaryk

University Brno, Kotlářská 2, Brno,

611 37, The Czech Republic

\begin{abstract}
We study vanishing at infinity solutions of a fourth-order nonlinear differential equation. We state sufficient and/or necessary conditions for the existence of the positive solution on the half-line $[0, \infty)$ which is vanishing at infinity and sufficient conditions ensuring that all eventually positive solutions are vanishing at infinity. We also discuss an oscillation problem.
\end{abstract}

\section{Introduction}

In this paper we study the fourth-order nonlinear differential equation

$$
x^{(4)}(t)+q(t) x^{\prime \prime}(t)+r(t)|x(t)|^{\lambda} \operatorname{sgn} x(t)=0 \quad\left(t \in \mathbb{R}_{+}\right),
$$

where $\lambda \geq 1, q \in C^{3}\left(\mathbb{R}_{+}\right), q(t)>0$ for large $t, r \in C\left(\mathbb{R}_{+}\right)$such that $r(t) \neq 0$ for large $t$ and $\mathbb{R}_{+}=[0, \infty)$.

Jointly with (1), we consider a more general equation

$$
x^{(4)}(t)+q(t) x^{\prime \prime}(t)+r(t) f(x(t))=0 \quad\left(t \in \mathbb{R}_{+}\right),
$$

where $f \in C^{3}(\mathbb{R})$ satisfies $f(u) u>0$ for $u \neq 0$, and the associated linear second-order equation

$$
h^{\prime \prime}(t)+q(t) h(t)=0 .
$$

By a solution of (1) we mean a function $x \in C^{4}\left[T_{x}, \infty\right), T_{x} \geq 0$, which satisfies (1) on $\left[T_{x}, \infty\right)$. A solution is said to be nonoscillatory if $x(t) \neq 0$ for large $t$; otherwise, it is said to be oscillatory. Observe that if $\lambda \geq 1$, according to [1, Theorem 11.5], all nontrivial solutions of (1) satisfy $\sup \{|x(t)|: t \geq T\}>0$ for $T \geq T_{x}$, on the contrary to the case $\lambda<1$, when nontrivial solutions satisfying $x(t) \equiv 0$ for large $t$ may exist.

Fourth-order differential equations have been investigated in detail during the last years. The periodic boundary value problem for the superlinear equation $x^{(4)}=g(x)+e(t)$ has been studied in [2]. In [3], the fourth-order linear eigenvalue problem, together with the nonlinear boundary value problem $x^{(4)}-f(t, x)=0$, has been investigated. Oscillatory properties of solutions for self-adjoint linear differential equations can be found in [4]. Equation (1) with $q(t) \equiv 0$ can be viewed as a prototype of even-order two-term differential equations, which are the main object of monographs $[1,5,6]$.

(c) 2013 Bartušek and Došlá; licensee Springer. This is an Open Access article distributed under the terms of the Creative Commons Attribution License (http://creativecommons.org/licenses/by/2.0), which permits unrestricted use, distribution, and reproduction in any medium, provided the original work is properly cited. 
Equation ( $\left.1^{\prime}\right)$ with $q(t) \equiv 1$ for $t \in \mathbb{R}_{+}$is a special case of higher-order differential equations investigated in [7]. Equation ( $\left(1^{\prime}\right)$ with $q$ near to a nonzero constant as $t \rightarrow \infty$ has been considered in [8] as a perturbation of the linear equation $y^{(4)}(t)+q(t) y^{\prime \prime}(t)=0$, and the existence of oscillatory solutions of $\left(1^{\prime}\right)$ has been proved. In [9], necessary and sufficient conditions for the existence of asymptotically linear solutions of $\left(1^{\prime}\right)$ have been given.

In the recent paper [10], the equation

$$
x^{(4)}(t)+k x^{\prime \prime}(t)+f(x(t))=0 \quad(t \in \mathbb{R})
$$

where $k \in \mathbb{R}, f(u) u>0$ for $u \neq 0$, and $f \in \operatorname{Lip}_{\text {loc }}(\mathbb{R})$ has been investigated and applications to the biharmonic PDE's can be found there. In particular, the so called homoclinics solutions, which are defined as nontrivial solutions $x$ such that $\lim _{t \rightarrow \pm \infty} x(t)=0$, are studied.

The goal of this paper is to investigate asymptotic problems associated with (1) and the asymptotic boundary condition

$$
x(t)>0 \quad \text { for large } t, \quad \lim _{t \rightarrow \infty} x(t)=0 .
$$

A solution $x$ of (1) satisfying (3) is said to be vanishing at infinity.

We start with the Kneser problem for (1). The Kneser problem is a problem concerning the existence of solutions of (1) subject to the boundary conditions on the half-line $[0, \infty)$

$$
x(0)=c>0, \quad(-1)^{i} x^{(i)}(t)>0 \quad \text { for } t \geq 0, i=0,1,2 .
$$

We establish necessary and/or sufficient conditions for the solvability of the boundary value problem (1), (3), (4). In the light of these results, as the second problem, we study when all eventually positive solutions $x$ of (1) are vanishing at infinity assuming that $\lambda>1$ and (2) is oscillatory. As a consequence, we give a bound for the set of all nonoscillatory solutions. Finally, we discuss when problem (1), (3) is not solvable and solutions to (1) are oscillatory.

A systematic analysis of solutions of (1) satisfying (3) is made according to whether (2) is nonoscillatory or oscillatory. If (2) is nonoscillatory, then the following approach will be used. Equation (1) can be rewritten as the two-term equation

$$
\left(h^{2}(t)\left(\frac{x^{\prime \prime}(t)}{h(t)}\right)^{\prime}\right)^{\prime}+h(t) r(t)|x(t)|^{\lambda} \operatorname{sgn} x(t)=0
$$

where $h$ is a positive solution of (2). According to [11], a solution $h$ of (2) is said to be a principal solution if $\int^{\infty} h^{-2}(t) d t=\infty$, and such a solution is determined uniquely up to a multiple constant. Since $q(t)>0$, every eventually positive solution of (2) is nondecreasing for large $t$. Hence there exists a principal solution $h$ of (2) such that $h(t)>0$ for $t \geq a \geq 0$ and

$$
\int_{a}^{\infty} \frac{1}{h^{2}(t)} d t=\infty, \quad \int_{a}^{\infty} h(t) d t=\infty
$$

Therefore, we can use the known results $[12,13]$ stated for systems of differential equations or in [14] for fourth order differential equations.

If (2) is oscillatory, then our approach is based on the choice of a suitable transformation. The main idea is based on a transformation of (1) to the fourth-order quasilinear equa- 
tion and the use of the estimates for positive solutions of such an equation on a compact interval stated in [15]. This, together with an energy function associated with (1), enables us to state an oscillation theorem. In the final section, some extensions of our results to $\left(1^{\prime}\right)$ are given.

\section{The Kneser problem}

In this section we present necessary and/or sufficient conditions for solvability of boundary value problem (1), (3), (4).

\subsection{Case $r(t)<0$}

Proposition 1 Let $\lambda \geq 1$, (2) be disconjugate on $[0, \infty)$, and $r(t)<0$ for $t \in \mathbb{R}_{+}$. Then boundary value problem (1), (4) is solvable for any $c>0$.

To prove this theorem, we use Chanturia's result [12, Theorem 1] for the system of differential equations

$$
\frac{d y}{d t}=f(t, y)
$$

where we restrict to the case that $f=\left(f_{1}, f_{2}, f_{3}, f_{4}\right): \mathbb{R}_{+} \times \mathbb{R}^{4} \rightarrow \mathbb{R}^{4}$ are continuous functions, $n=4, m=1, l=2, a=1$ and $r=c$. Then this result reads as follows.

Theorem A ([12]) Let there exist $c>0$ such that

$$
\begin{gathered}
f_{i}\left(t, y_{1}, y_{2}, y_{3}, y_{4}\right) \leq 0 \quad(i=1, \ldots, 4) \\
\text { for } t \in \mathbb{R}_{+}, y_{1} \in[0, c), y_{i} \in \mathbb{R}_{+}(i=2,3,4) . \text { Suppose } \\
-f_{i}\left(t, y_{1}, y_{2}, y_{3}, y_{4}\right) \geq \varphi_{i}\left(t, y_{i+1}\right) \quad(i=1,2,3) \\
-\sum_{i=2}^{4} f_{i}\left(t, y_{1}, y_{2}, y_{3}, y_{4}\right) \leq \psi(t) \omega\left(\sum_{i=2}^{4} y_{i}\right)
\end{gathered}
$$

for $t \in[0,1], y_{1} \in[0, c], y_{i} \in \mathbb{R}_{+}(i=2,3,4)$, where functions $\varphi_{i}:[0,1] \times \mathbb{R}_{+} \rightarrow \mathbb{R}_{+}(i=1,2,3)$ are continuous and nondecreasing in the second argument such that

$$
\begin{aligned}
& \liminf _{x \rightarrow \infty} \int_{0}^{1} \varphi_{1}(t, x) d t>c, \\
& \lim _{x \rightarrow \infty} \int_{0}^{1} \varphi_{i}(t, x) d t=\infty \quad(i=2,3),
\end{aligned}
$$

$\psi:[0,1] \rightarrow \mathbb{R}_{+}$is a continuous function and $\omega: \mathbb{R}_{+} \rightarrow(0, \infty)$ is a continuous nondecreasing function such that

$$
\int_{0}^{\infty} \frac{d u}{\omega(u)}=\infty
$$

Then, for any $x_{0} \in[0, c]$, system (7) has a solution satisfying

$$
y_{1}(0)=x_{0}, \quad y_{i}(t) \geq 0, \quad y_{i}^{\prime}(t) \leq 0 \quad \text { for } t \in \mathbb{R}_{+}(i=1,2,3,4) .
$$


Proof of Proposition 1 Assume $r(t)<0$ for $t \in \mathbb{R}_{+}$. Since (2) is disconjugate, it has a positive solution $h$ on $\mathbb{R}_{+}$, and (1) can be written as (5) where $h(t) r(t)<0$ on $\mathbb{R}_{+}$. Let $x$ be a solution of (5) and denote

$$
y_{1}(t)=x(t), \quad y_{2}(t)=-y_{1}^{\prime}(t), \quad y_{3}(t)=-\frac{1}{h(t)} y_{2}^{\prime}(t), \quad y_{4}(t)=-h^{2}(t) y_{3}^{\prime}(t)
$$

Then (5) is equivalent to the system

$$
\left\{\begin{array}{l}
y_{1}^{\prime}(t)=-y_{2}(t), \\
y_{2}^{\prime}(t)=-h(t) y_{3}(t), \\
y_{3}^{\prime}(t)=-\frac{1}{h^{2}(t)} y_{4}(t), \\
y_{4}^{\prime}(t)=-h(t)|r(t)|\left|y_{1}(t)\right|^{\lambda}(t) \operatorname{sgn} y_{1}(t) \quad\left(t \in \mathbb{R}_{+}\right) .
\end{array}\right.
$$

Let $c>0$ be from (4). We apply Theorem A choosing

$$
\varphi_{1}(t, x)=x, \quad \varphi_{i}(t, x)=k_{i} x, \quad i=2,3, \quad \psi(t)=k_{4}, \quad \omega(u)=c^{\lambda}+u,
$$

where $k_{2}=\min _{t \in[0,1]} h(t), k_{3}=\min _{t \in[0,1]} 1 / h^{2}(t)$ and $k_{4}=\max _{t \in[0,1]}\left[h(t)+1 / h^{2}(t)+h(t) r(t)\right]$. By this result, system (10) has a solution such that

$$
y_{1}(0)=c>0, \quad y_{i}(t) \geq 0, \quad y_{i}^{\prime}(t) \leq 0 \quad \text { for } t \geq 0, \quad i=1,2,3,4 \text {. }
$$

Since $\lambda \geq 1$, system (10) has no solutions such that $y_{i} \equiv 0$ for some $i=1,2,3,4$ and large $t$; see [16] or [17, Lemma 2, Theorem 2]. Thus, for any $c>0$, equation (1) has a solution $x$ such that $x(0)=c, x(t)=y_{1}(t)>0, x^{\prime}(t)=-y_{2}(t)<0$ and $x^{\prime \prime}(t)=h(t) y_{3}(t)>0$ for $t \geq 0$.

Now we state conditions for the existence of a solution for problem (1), (3), (4).

Theorem 1 Let $\lambda \geq 1, r(t)<0$ and

$$
q(t)(t+1)^{2} \leq \frac{1}{4}
$$

on $\mathbb{R}_{+}$. If

$$
\int_{0}^{\infty} t^{2}|r(t)| d t=\infty
$$

then problem (1), (3), (4) is solvable for any $c>0$.

In addition, if

$$
\int_{0}^{\infty} t q(t) d t<\infty
$$

then the condition

$$
\int_{0}^{\infty} t^{3}|r(t)| d t=\infty
$$

is necessary and sufficient for the solvability of problem (1), (3), (4). 
For the proof, the following lemma will be needed.

Lemma 1 Consider system (10) on $[a, \infty)(a \geq 0)$, where $h(t)>0$ for $t \geq a$ and $h$ is a principal solution of (2). Let $y=\left(y_{1}, y_{2}, y_{3}, y_{4}\right)$ be a solution of $(10)$ such that $y_{i}(t) \geq 0$ and $y_{i}^{\prime}(t) \leq 0$ for $i=1,2,3,4$, and $t \geq a$. Then $\lim _{t \rightarrow \infty} y_{i}(t)=0$ for $i=2,3,4$, and if

$$
\int_{a}^{\infty} h(t)|r(t)| \int_{a}^{t} \frac{1}{h^{2}(s)} \int_{a}^{s} h(\tau) \tau d \tau d s d t=\infty
$$

then $\lim _{t \rightarrow \infty} y_{1}(t)=0$, too. Vice versa, if $\lambda \geq 1$ and $\lim _{t \rightarrow \infty} y_{1}(t)=0$, then (15) holds.

Proof In view of the monotonicity of $y_{i}$, there exist $\lim _{t \rightarrow \infty} y_{i}(t)=y_{i}(\infty) \geq 0, i=1,2,3,4$. Since $h$ is the principal solution, (6) holds, and integrating the first three equations in (10) from $a$ to $t$, we get $y_{i}(\infty)=0$ for $i=2,3,4$. Now integrating (10) from $t$ to $\infty$, we have

$$
\begin{aligned}
& y_{2}(t)=\int_{t}^{\infty} h(s) y_{3}(s) d s, \\
& y_{3}(t)=\int_{t}^{\infty} \frac{1}{h^{2}(t)} y_{4}(s) d s, \\
& y_{4}(t)=\int_{t}^{\infty} h(s)|r(s)| y_{1}^{\lambda}(s) d s .
\end{aligned}
$$

Let (15) hold and assume, by contradiction, that $y_{1}(\infty)>0$. Then

$$
y_{1}(a)-y_{1}(t) \geq y_{1}^{\lambda}(\infty) \int_{a}^{t} \int_{s}^{\infty} h(u) \int_{u}^{\infty} \frac{1}{h^{2}(v)} \int_{v}^{\infty} h(\tau)|r(\tau)| d \tau d v d u d s
$$

Letting $t \rightarrow \infty$ and using the change of the order of integration, we get a contradiction with the boundedness of $y_{1}$. This proves that $y_{1}(\infty)=0$.

Let the integral in (15) be convergent and assume, by contradiction, that $y_{1}(\infty)=0$. Then we have

$$
y_{1}(t)=\int_{t}^{\infty} \int_{s}^{\infty} h(u) \int_{u}^{\infty} \frac{1}{h^{2}(v)} \int_{v}^{\infty} h(\tau)|r(\tau)| y_{1}^{\lambda}(\tau) d \tau d v d u d s
$$

so

$$
y_{1}(t) \leq y_{1}^{\lambda}(t) \int_{t}^{\infty} \int_{s}^{\infty} h(u) \int_{u}^{\infty} \frac{1}{h^{2}(v)} \int_{v}^{\infty} h(\tau)|r(\tau)| d \tau d v d u d s
$$

Since $\lambda \geq 1$, then using the change of the order of integration, we get a contradiction for large $t$. This proves that $y_{1}(\infty)>0$.

Proof of Theorem 1 In view of (11), (2) is disconjugate on $\mathbb{R}_{+}$. By Proposition 1, equation (1) has a solution $x$ satisfying (4). Therefore, system (10) has a solution such that $y_{i}(t)>0$ and $y_{i}^{\prime}(t)<0$ for $t \in \mathbb{R}_{+}$. Choose $h$ in (10) as a principal solution of (2). The Euler equation

$$
\tilde{h}^{\prime \prime}+\frac{1}{4(t+1)^{2}} \tilde{h}=0 \quad\left(t \in \mathbb{R}_{+}\right)
$$


is the majorant of (2) on $\mathbb{R}_{+}$and has the principal solution $\tilde{h}(t)=\sqrt{t+1}$. By the comparison theorem, for the minimal solution of the Riccati equation related to (2) and (17), we have

$$
0<\frac{h^{\prime}(t)}{h(t)} \leq \frac{\tilde{h}^{\prime}(t)}{\tilde{h}(t)}
$$

for large $t$; see, e.g., [11]. Thus there exists $\ell>0$ such that $\ell \leq h(t) \leq \ell \sqrt{t+1}$ for $t \geq 0$. Assume (12). Then (15) holds, and by Lemma 1 a solution $x$ satisfies (3).

Assume (13). Then the principal solution $h$ of (2) satisfies $h(t) \sim \ell$ for large $t$ (see, e.g., [11]). Hence, condition (15) reads as (14), and by Lemma 1 this condition is equivalent to the property (3).

As a consequence of Lemma 1, we get the following result.

Corollary 1 Let (2) be disconjugate on $[0, \infty)$, and $r(t)<0$ for $t \in \mathbb{R}_{+}$. Then any solution $x$ of (1) satisfying

$$
x(0)=c>0, \quad x(t)>0, \quad t \geq 0, \quad \lim _{t \rightarrow \infty} x(t)=0
$$

is a solution of the Kneser problem, i.e., $(-1)^{i} x^{(i)}(t)>0$ for $t \geq 0$ and $i=1,2$.

Proof Let $h$ be a positive solution on $\mathbb{R}_{+}$satisfying (6), and let $x$ be a solution of (1) satisfying (18). Then $y=\left(y_{1}, y_{2}, y_{3}, y_{4}\right)$, where $y_{1}$ are defined by (9), is a solution of system (10). Since $y_{1}(t)>0$ for $t \geq 0$ and (6) holds, we have by the Kiguradze lemma (see, e.g., [1]) that either $y_{i}^{\prime}(t)>0$ or $y_{i}^{\prime}(t)<0$ for $i=1,2,3$ and large $t$, say for $t \geq a \geq 0$. Since $x$ is positive and tends to zero, we have $y_{1}^{\prime} \leq 0$ for $t \geq a$, so also $y_{i}^{\prime} \leq 0(i=2,3)$ for $t \geq a$. By Lemma 1 , we get $y_{i}^{\prime}(\infty)=0(i=2,3)$ for $t \geq a$. Since $x(t)>0$ for $t \geq 0$, we have $y_{4}^{\prime}(t)<0$ for $t \geq 0$ and $y_{4}$ is positive and decreasing on $\mathbb{R}_{+}$. Hence, proceeding by the same argument, $y_{i}(i=2,3)$ is positive and decreasing on $\mathbb{R}_{+}$. Now the conclusion follows from (9).

\subsection{Case $r(t)>0$}

First we show that the sign condition posed on $r$ is necessary for the solvability of problem (1), (4).

A function $g$, defined in a neighborhood of infinity, is said to change sign if there exists a sequence $\left\{t_{k}\right\} \rightarrow \infty$ such that $g\left(t_{k}\right) g\left(t_{k+1}\right)<0$.

Theorem 2 Let $r(t)>0$ for large $t$. Then problem (1), (4) has no solution and the following hold:

(a) If (2) is nonoscillatory, then every nonoscillatory solution $x$ of (1) satisfies $x(t) x^{\prime}(t)>0$ and $x^{\prime \prime}$ is of one sign for large $t$.

(b) If (2) is oscillatory, then every nonoscillatory solution $x$ of (1) satisfies either $x(t) x^{\prime \prime}(t) \leq 0$, or $x^{\prime \prime}(t)$ changes sign. In addition, if a solution $x$ satisfies (3), then $x^{\prime \prime}$ changes sign.

Proof Claim (a). Let $x$ be a positive solution of (1) on $[a, \infty)$, or, equivalently, of (5) on $[a, \infty)$, where $h$ satisfies (6). Denote

$$
y_{1}(t)=x(t), \quad y_{2}(t)=y_{1}^{\prime}(t), \quad y_{3}(t)=\frac{1}{h(t)} y_{2}^{\prime}(t), \quad y_{4}(t)=h^{2}(t) y_{3}^{\prime}(t) .
$$


Then (5) is equivalent to the system

$$
\left\{\begin{array}{l}
y_{1}^{\prime}(t)=y_{2}(t), \\
y_{2}^{\prime}(t)=h(t) y_{3}(t), \\
y_{3}^{\prime}(t)=\frac{1}{h^{2}(t)} y_{4}(t), \\
y_{4}^{\prime}(t)=-h(t) r(t) y_{1}^{\lambda}(t) \operatorname{sgn} y_{1}(t) \quad(t \geq a) .
\end{array}\right.
$$

We have $y_{1}(t)>0$ for $t \geq a$. Assume by contradiction that $y_{2}(t)<0$ for $t \geq a$. Let $y_{3}(t)>0$ and $y_{4}(t)<0$. Since $y_{4}$ is nonincreasing, $y_{4}(t) \leq y_{4}(a)<0$ and

$$
y_{3}(t)-y_{3}(a) \leq y_{4}(a) \int_{a}^{t} 1 / h^{2}(s) d s .
$$

Letting $t \rightarrow \infty$, we get a contradiction with the positiveness of $y_{3}$. The remaining case $y_{3}(t)<0$ can be eliminated in a similar way using (6). Observe that system (20) is a special case of the Emden-Fowler system investigated in [13], and the proof follows also from [13, Lemma 2.1].

Claim (b). Without loss of generality, suppose that $r(t)>0$ for $t \geq T$ and there exists a solution $x$ of (1) such that $x(t)>0$ and $x^{\prime \prime}(t) \geq 0$ on $[T, \infty), T \geq 0$. Borůvka [18] proved that if (2) is oscillatory, then there exists a function $\alpha \in C^{3}[T, \infty)$, called a phase function, such that $\alpha^{\prime}(t)>0$ and

$$
\frac{3\left(\alpha^{\prime \prime}(t)\right)^{2}}{4\left(\alpha^{\prime}(t)\right)^{4}}-\frac{\alpha^{\prime \prime \prime}(t)}{2\left(\alpha^{\prime}(t)\right)^{3}}+\frac{q(t)}{\alpha^{\prime 2}(t)}=1
$$

Using this result, we can consider the change of variables

$$
s=\alpha(t), \quad x^{\prime \prime}(t)=\frac{1}{\sqrt{\alpha^{\prime}(t)}} X(s) \quad\left(\cdot=\frac{d}{d s}\right)
$$

for $t \in[T, \infty), s \in\left[T^{*}, \infty\right), T^{*}=\alpha(T)$. Thus, $t=\alpha^{-1}(s)$ and

$$
\begin{aligned}
& x^{\prime \prime \prime}(t)=-\frac{1}{2}\left(\alpha^{\prime}(t)\right)^{-\frac{3}{2}} \alpha^{\prime \prime}(t) X(s)+\left(\alpha^{\prime}(t)\right)^{1 / 2} \dot{X}(s), \\
& x^{(4)}(t)=-\frac{1}{2}\left(\alpha^{\prime}(t)\right)^{-\frac{3}{2}} \alpha^{\prime \prime \prime}(t) X(s)+\frac{3}{4}\left(\alpha^{\prime}(t)\right)^{-\frac{5}{2}}\left(\alpha^{\prime \prime}(t)\right)^{2} X(s)+\left(\alpha^{\prime}(t)\right)^{\frac{3}{2}} \ddot{X}(s) .
\end{aligned}
$$

Substituting into (1), we obtain the second-order equation

$$
\begin{aligned}
& \ddot{X}(s)+X(s)\left(\frac{3}{4}\left(\alpha^{\prime}(t)\right)^{-4}\left(\alpha^{\prime \prime}(t)\right)^{2}-\frac{1}{2}\left(\alpha^{\prime}(t)\right)^{-3} \alpha^{\prime \prime \prime}(t)+q(t)\left(\alpha^{\prime}(t)\right)^{-2}\right) \\
& \quad+\left(\alpha^{\prime}(t)\right)^{-\frac{3}{2}} r(t) x^{\lambda}(t)=0 .
\end{aligned}
$$

From here and (21), we obtain

$$
\ddot{X}(s)+X(s)=-\left(\alpha^{\prime}(t)\right)^{-\frac{3}{2}} r(t) x^{\lambda}(t)<0 .
$$

Since $X^{\prime \prime}(t) \geq 0$, (22) yields $X(s) \geq 0$ and so $\ddot{X}(s)<0$, that is, $\dot{X}$ is decreasing. If there exists $s_{1} \geq T$ such that $\dot{X}\left(s_{1}\right)<0, X$ becomes eventually negative, which is a contradiction. Then 
$\dot{X}(s) \geq 0$ and $X(s)$ is nondecreasing. Let $T_{1} \geq T^{*}$ be such that $X(s)>0$ on $\left[T_{1}, \infty\right)$. Thus, using (23) we obtain

$$
\dot{X}(s)-\dot{X}\left(T_{1}\right)=\int_{T_{1}}^{s} \ddot{X}(u) d u \leq-\int_{T_{1}}^{s} X(u) d u \leq-X\left(T_{1}\right)\left(s-T_{1}\right) .
$$

Hence, $\lim _{s \rightarrow \infty} \dot{X}(s)=-\infty$, which contradicts the nonnegativity of $\dot{X}(s)$. Finally, the case $X(s) \equiv 0$ on $\left[T^{*}, \infty\right)$ cannot occur, because if $x^{\prime \prime}(t) \equiv 0$ on $[T, \infty)$, then from (1) and $r(t)>0$, we have $x \equiv 0$ on $[T, \infty)$, which is a contradiction.

Finally, let $x$ be a positive solution of (1) satisfying (3). Then $x^{\prime \prime}$ is either oscillatory or $x^{\prime \prime}(t)<0$ for large $t$. Assume $x^{\prime \prime}(t)<0$ on some $J=[T, \infty)$, then $x^{\prime}$ is decreasing and either $x^{\prime}(t) \geq 0$ or $x^{\prime}(t)<0$ for large $t$. If $x^{\prime}(t) \geq 0$ for large $t$, then we get a contradiction with (3). If $x^{\prime}(t)<0$, then $x^{\prime}(t) \leq x^{\prime}\left(T_{1}\right)<0$ for $t \geq T_{1} \geq T$ and $x$ becomes negative for large $t$. Hence $x^{\prime \prime}$ must be oscillatory.

For $\lambda>1$, the analogous result to Theorem 1 is the following oscillation result.

Proposition 2 Let $\lambda>1, r(t)>0$ for large t. Assume either (11) for large t, (12), or (13), (14). Then all the solutions of (1) are oscillatory.

Proof Let $x$ be a solution of (1) and $h$ be the principal solution of (2). Then $y=\left(y_{1}, y_{2}, y_{3}, y_{4}\right)$, where $y_{i}$ are given by (19), is a solution of system (20). Proceeding by the similar way as in the proof of Theorem 1, we have that (15) holds. Using the change of the order of integration in (15), we can check that conditions of Theorem 4.3 in [13] applied to system (20) are verified. Hence by this result all the solutions of (20) are oscillatory, which gives the conclusion.

The following result follows from [7, Theorem 1.5] and completes Proposition 2 in the case when (2) is oscillatory.

Proposition 3 Let $\lambda>1, q(t) \equiv 1$ and $r(t)>0$ for $t \in \mathbb{R}_{+}$. Then the condition $\int^{\infty} \operatorname{tr}(t) d t=$ $\infty$ is necessary and sufficient for every solution of (1) to be oscillatory.

In the light of these results, in the sequel, we study asymptotic and oscillation problems to (1) when (2) is oscillatory.

\section{Vanishing at infinity solutions}

In this section we study when all nonoscillatory solutions of (1) are vanishing at infinity.

Theorem 3 Let $\lambda>1$ and (2) be oscillatory. Assume that $q(t) \geq K t^{-2}$ for large $t$ and some $K>0$, the functions

$$
\frac{q^{\prime}}{q^{3 / 2}}, \quad \frac{q^{\prime \prime}}{q^{2}}, \quad \frac{q^{\prime \prime \prime}}{q^{5 / 2}} \quad \text { are bounded on } \mathbb{R}_{+},
$$

and

$$
\frac{|r(t)|}{q^{2}(t)} \uparrow \infty \quad \text { as } t \rightarrow \infty .
$$

Then any eventually positive solution of (1) is vanishing at infinity. 
The proof of Theorem 3 is based on the following auxiliary results.

Consider the fourth-order quasi-linear differential equation

$$
y^{(4)}(s)+\sum_{i=1}^{3} Q_{i}(s) y^{(i)}(s)+R(s)|y|^{\lambda}(s) \operatorname{sgn} y(s)=0,
$$

where $Q_{i}$ and $R$ are continuous functions on [0, $\infty$ ). In [15, Theorem 2.4], the following uniform estimate for positive solutions of (26) with a common domain was proved.

Proposition 4 ([15, Theorem 3.4, Corollary 3.6]) Assume $\lambda>1$. Let y be a positive solution of (26) defined on $[0, b]$ and

$$
|R(s)| \geq r_{*}, \quad\left|Q_{i}(s)\right| \leq Q^{4-i}, \quad i=1,2,3,
$$

on $[0, b]$ for some constants $r_{*}>0$ and $Q>0$. Then there exists a positive constant $M=M(\lambda)$ such that

$$
y(s) \leq M r_{*}^{-\frac{1}{\lambda-1}} \delta^{-\frac{4}{\lambda-1}}(s) \quad \text { for } s \in[0, b]
$$

where

$$
\delta(s)=\min \{s, \alpha, b-s\}, \quad \alpha=2^{-19} Q^{-1} .
$$

Remark 1 In [15, Theorem 3.4] the constant $M$ is explicitly calculated.

Lemma 2 Let $\lambda>1$. Assume that (27) holds on $[0, \infty)$. Then any positive solution of $(26)$ defined on $[0, \infty)$ satisfies

$$
y(s) \leq M r_{*}^{-\frac{1}{\lambda-1}} \alpha^{-\frac{4}{\lambda-1}} \quad \text { for } s \in[\alpha, \infty),
$$

where $\alpha$ and $M$ are constants from Proposition 4.

Proof Let $b \geq \alpha$. By Proposition 4, applied on $[0, b]$, we have $\delta=\delta(s) \equiv \alpha$ for $s \in[\alpha, b-\alpha]$ and

$$
y(s) \leq M r_{*}^{-\frac{1}{\lambda-1}} \alpha^{-\frac{4}{\lambda-1}} \quad \text { for } s \in[\alpha, b-\alpha] .
$$

Letting $b \rightarrow \infty$, we get (29).

The next lemma describes the transformation between solutions of (1) and a certain quasi-linear equation.

Lemma 3 Let $q(t)>0$ on $[a, \infty)$ be such that

$$
\int_{a}^{\infty} \sqrt{q(t)} d t=\infty
$$


and consider the transformation

$$
s=\int_{a}^{t} \sqrt{q(t)} d t, \quad x(t)=y(s), \quad \cdot=\frac{d}{d s} .
$$

Then $x=x(t)$ is a solution of equation (1) on $[a, \infty)$ if and only if $y=y(s)$ is a solution of the equation

$$
\begin{aligned}
& \frac{d^{4} y}{d s^{4}}+3 \frac{q^{\prime}(t)}{q^{3 / 2}(t)} \frac{d^{3} y}{d s^{3}}+\left[2 \frac{q^{\prime \prime}(t)}{q^{2}(t)}-\frac{\left(q^{\prime}(t)\right)^{2}}{4 q^{3}(t)}+1\right] \ddot{y} \\
& +\left[\frac{q^{\prime \prime \prime}(t)}{2 q^{5 / 2}(t)}-\frac{3}{4} \frac{q^{\prime}(t) q^{\prime \prime}(t)}{q^{7 / 2}(t)}+\frac{3}{8} \frac{\left(q^{\prime}(t)\right)^{3}}{q^{9 / 2}(t)}+\frac{q^{\prime}(t)}{2 q^{3 / 2}(t)}\right] \dot{y} \\
& +\frac{r(t)}{q^{2}(t)}|y|^{\lambda} \operatorname{sgn} y=0 \quad \text { on }[0, \infty),
\end{aligned}
$$

where $t=t(s)$ is the inverse function to $s=s(t)$.

Proof We have

$$
\begin{aligned}
x^{\prime}(t)= & \dot{y}(s) \sqrt{q(t)}, \quad x^{\prime \prime}(t)=\ddot{y}(s) q(t)+\frac{\dot{y}(s) q^{\prime}(t)}{2 \sqrt{q(t)}}, \\
x^{\prime \prime \prime}(t)= & \frac{d^{3} y(s)}{d s^{3}} q^{3 / 2}(t)+\frac{3}{2} \ddot{y}(s) q^{\prime}(t)+\dot{y}(s)\left[\frac{q^{\prime \prime}(t)}{2 \sqrt{q(t)}}-\frac{\left(q^{\prime}(t)\right)^{2}}{4 q^{3 / 2}(t)}\right], \\
x^{(4)}(t)= & \frac{d^{4} y(s)}{d s^{4}} q^{2}(t)+3 \frac{d^{3} y}{d s^{3}} q^{\prime}(t) \sqrt{q(t)}+\ddot{y}(s)\left[2 q^{\prime \prime}(t)-\frac{\left(q^{\prime}(t)\right)^{2}}{4 q(t)}\right] \\
& +\dot{y}(s)\left[\frac{q^{\prime \prime \prime}(t)}{2 q^{1 / 2}(t)}-\frac{3}{4} \frac{q^{\prime}(t) q^{\prime \prime}(t)}{q^{3 / 2}(t)}+\frac{3}{8} \frac{\left(q^{\prime}(t)\right)^{3}}{q^{5 / 2}(t)}\right] .
\end{aligned}
$$

Substituting into (1), we get the conclusion.

Proof of Theorem 3 Let $x$ be a positive solution of (1) on $I=[a, \infty)(a>0)$. Suppose, for simplicity, that $q(t) \geq K t^{-2}$ for $t \geq a$. Let

$$
\left|q^{\prime}(t)\right| q^{-\frac{3}{2}}(t) \leq C_{1}, \quad\left|q^{\prime \prime}(t)\right| q^{-2}(t) \leq C_{2}, \quad\left|q^{\prime \prime \prime}(t)\right| q^{-\frac{5}{2}}(t) \leq C_{3}
$$

on $[0, \infty)$ for some positive constants $C_{1}, C_{2}, C_{3}$ and

$$
Q=\max \left\{3 C_{1},\left(2 C_{2}+\frac{1}{4} C_{1}^{2}+1\right)^{1 / 2},\left(\frac{C_{3}}{2}+\frac{3}{4} C_{1} C_{2}+\frac{3}{8} C_{1}^{3}+\frac{C_{1}}{2}\right)^{1 / 3}\right\} .
$$

Denote

$$
\alpha=2^{-19} Q^{-1}, \quad \bar{a}=\exp (\alpha / K) .
$$

Define the function $t^{*}=t^{*}(t)$ such that

$$
\int_{t^{*}}^{t} \sqrt{q(s)} d s=\alpha
$$


for $t \geq \bar{a}$. Then, according to $q(t) \geq K t^{-2}$, we have

$$
t^{*} \geq t \exp (-\alpha / K) \geq 1
$$

Let $r / q^{2}$ be nondecreasing on $[\tilde{a}, \infty)$ and put $T \geq \max \{a, \bar{a}, \tilde{a}\}$. Choose $t_{0} \in[T, \infty)$ arbitrarily fixed. Since $q(t) \geq K t^{-2}$, we can consider the transformation from Lemma 3 with $a=t^{*}\left(t_{0}\right)$, i.e.,

$$
s=\int_{t^{*}\left(t_{0}\right)}^{t} \sqrt{q(\tau)} d \tau, \quad x(t)=y(s), \quad \cdot=\frac{d}{d s} .
$$

Then equation (1) is transformed into equation (30) which is a quasilinear equation of the form (26), where

$$
\left|Q_{1}(s)\right| \leq Q^{3}, \quad\left|Q_{2}(s)\right| \leq Q^{2}, \quad\left|Q_{3}(s)\right| \leq Q, \quad R(s)=\frac{r(t(s))}{q^{2}(t(s))}
$$

and $Q$ is defined by (31) and (32). Choose $t_{0} \geq T$ arbitrarily. We apply Lemma 2 to equation (30) with

$$
r_{*}=\min _{s \geq 0} \frac{|r(t(s))|}{q^{2}(t(s))}=\frac{\left|r\left(t^{*}\left(t_{0}\right)\right)\right|}{q^{2}\left(t^{*}\left(t_{0}\right)\right)} .
$$

Hence estimate (29) with $s=\alpha$ reads as

$$
x\left(t_{0}\right)=y(\alpha) \leq C\left(\frac{q^{2}\left(t^{*}\left(t_{0}\right)\right)}{\left|r\left(t^{*}\left(t_{0}\right)\right)\right|}\right)^{\frac{1}{\lambda-1}},
$$

where $C=M \alpha^{-\frac{4}{\lambda-1}}$. Letting $t_{0} \rightarrow \infty$, we have by (35) that $t^{*}\left(t_{0}\right) \rightarrow \infty$ and the conclusion follows from (25) and (37).

From the proof of Theorem 3, we get the estimate for the set of all nonoscillatory solutions of (1) which will be used in the next section.

Corollary 2 Let $\lambda>1, \lim _{t \rightarrow \infty} q(t)=\infty,(24)$ and (25) hold. Then, for any $\varepsilon>0$, there exists a positive constant $C=C(\lambda, q(t), \varepsilon)$ and $T \geq 0$ such that every nonoscillatory solution $x$ of (1) satisfies

$$
|x(t)| \leq C\left(\frac{q^{2}(t-\varepsilon)}{|r(t-\varepsilon)|}\right)^{\frac{1}{\lambda-1}} \quad \text { for } t \geq T .
$$

Proof Let $\varepsilon>0$ be fixed and let $T \geq 0$ be such that

$$
q(t) \geq \frac{\alpha^{2}}{\varepsilon^{2}} \quad \text { for } t \geq T,
$$

where $\alpha$ is given by (33). Let $t \geq T$ be fixed. Using estimate (37) with $t_{0}=t \geq T$, we have

$$
x(t) \leq C\left(\frac{q^{2}\left(t^{*}(t)\right)}{\left|r\left(t^{*}(t)\right)\right|}\right)^{\frac{1}{\lambda-1}} \quad(t \geq T),
$$


where $t^{*}$ is given by (34), i.e.,

$$
\alpha=\int_{t^{*}(t)}^{t} \sqrt{q(\tau)} d \tau \geq \frac{\alpha}{\varepsilon}\left(t-t^{*}(t)\right)
$$

Therefore $t^{*}(t) \geq t-\varepsilon$ and estimate (38) follows from (25) and (39).

Example 1 Consider the equation

$$
x^{(4)}(t)+(t+1) x^{\prime \prime}(t)-\left(24(t+1)^{4}+2(t+1)^{7}\right) x^{9}(t)=0 \quad\left(t \in \mathbb{R}_{+}\right) .
$$

Then $r / q^{2} \rightarrow \infty$ and by Theorem 3 all eventually positive solutions are vanishing at infinity. One can check that $x=\frac{1}{t+1}$ is such a solution of (40).

Open problem It is an open problem to find conditions for the solvability of boundary value problem (1), (3), (4) in case $r(t)<0$ and (2) is oscillatory.

In view of Theorem 2, Corollary 2 and Proposition 1, it is a question whether (1) can have vanishing at infinity solutions in case $r(t)>0$ and (2) is oscillatory.

In the next section, we show that under certain additional assumptions the answer is negative.

\section{Oscillation}

Here we consider (1) in case $r(t)>0$ for large $t$. When (2) is nonoscillatory, we have established the oscillation criterion in Proposition 2. When (2) is oscillatory, the following oscillation theorem holds.

Theorem 4 Let $\lambda>1, r(t)>0$ and assumptions (24), (25) hold. Assume

$$
\lim _{t \rightarrow \infty} q(t)=\infty, \quad q^{\prime}(t) \geq 0, \quad q^{\prime \prime}(t) \geq 0 \quad \text { for large } t
$$

and

$$
\limsup _{t \rightarrow \infty} q^{1+\sigma}(t)\left(\frac{q^{2}(t-\varepsilon)}{r(t-\varepsilon)}\right)^{\frac{1}{\lambda-1}}<\infty
$$

for some $\varepsilon>0$ and $\sigma>0$. Then problem (1), (3) is not solvable and all the solutions of (1) are oscillatory.

Proof Suppose that (25) holds on $[a, \infty)$. First, observe that the assumption (42) implies that

$$
\int_{0}^{\infty} q^{\prime}(t)\left(\frac{q^{2}(t-\epsilon)}{r(t-\epsilon)}\right)^{\frac{1}{\lambda-1}} d t<\infty .
$$

Indeed, putting $H(t)=\left(q^{2}(t) / r(t)\right)^{\frac{1}{\lambda-1}}$, we have

$$
\int_{a}^{t} q^{\prime}(s) H(s) d s<H(a) q^{1+\sigma}(t) \int_{a}^{t} \frac{q^{\prime}(s)}{q^{1+\sigma}(s)} d t,
$$


and thus, in view of (42), we get (43). Consider a solution $x$ of (1) such that $x(t)>0$ for $t \geq t_{0} \geq 0$. According to Corollary 2 , there exists $\bar{t}_{0}$ such that

$$
0<x(t) \leq C\left(\frac{q^{2}(t-\epsilon)}{r(t-\epsilon)}\right)^{\frac{1}{\lambda-1}} \quad \text { for } t \geq \bar{t}_{0} \geq t_{0}
$$

and in view of (25) we get $\lim _{t \rightarrow \infty} x(t)=0$. Consider the function

$$
F(t)=-x^{\prime \prime \prime}(t)-q(t) x^{\prime}(t)+q^{\prime}(t) x(t) .
$$

Then

$$
F^{\prime}(t)=r(t) x^{\lambda}(t)+q^{\prime \prime}(t) x(t)
$$

and in view of (41) the function $F$ is increasing for large $t$. Hence, there exists $t_{2} \geq t_{1}$ such that either

$$
F(t)<0 \quad \text { for } t \geq t_{2},
$$

or

$$
F(t) \geq F\left(t_{2}\right)>0 \quad \text { for } t \geq t_{2} .
$$

According to Theorem 2(b), $x^{\prime \prime}$ oscillates. Define by $\left\{\tau_{k}\right\}_{k=1}^{\infty}$ an increasing sequence of zeros of $x^{\prime \prime}$ tending to $\infty$ with $\tau_{1} \geq t_{2}$.

Define

$$
Z(t)=-x^{\prime \prime}(t)-q(t) x(t)-2 \int_{t}^{\infty} q^{\prime}(s) x(s) d s .
$$

In view of (44) and (43) the function $Z$ is well defined and

$$
Z^{\prime}(t)=-x^{\prime \prime \prime}(t)-q(t) x^{\prime}(t)+q^{\prime}(t) x(t)=F(t)
$$

on $\left[t_{0}, \infty\right)$. Moreover, we have from (42), (44) and (47)

$$
\lim _{k \rightarrow \infty} Z\left(\tau_{k}\right)=0
$$

If (45) holds, then $Z^{\prime}(t)<0$ and because

$$
Z\left(\tau_{1}\right)=-q\left(\tau_{1}\right) x\left(\tau_{1}\right)-2 \int_{\tau_{1}}^{\infty} q^{\prime}(s) x(s) d s<0,
$$

we get $Z(t) \leq Z\left(\tau_{1}\right)<0$ for $t \geq \tau_{1}$. This is a contradiction with (49), so (45) is impossible. If (46) holds, then $Z^{\prime}(t)>0$ and $\lim _{t \rightarrow \infty} Z(t)=\infty$. This is again a contradiction with (49), so also this case is impossible. 
Example 2 Consider the equation

$$
x^{(4)}(t)+\frac{c}{t^{2}} x^{\prime \prime}(t)+\sigma|x|^{3}(t) \operatorname{sgn} x(t)=0 \quad\left(t \in \mathbb{R}_{+}\right),
$$

where $\sigma= \pm 1$. If $\sigma=-1$ and $c \in(0,1 / 4)$, then by Theorem 1 this equation has a solution satisfying (3) and (4). If $\sigma= \pm 1$ and $c>1 / 4$, then by Theorem 3 any nonoscillatory solution (if any) satisfies (3).

\section{Extensions}

As it was mentioned in [10], a certain nonlinear PDE leads to the fourth-order equation with the exponential nonlinearity. In the sequel, we show that the results of this paper can be extended to the nonlinear equation

$$
x^{(4)}(t)+q(t) x^{\prime \prime}(t)+r(t) f(x(t))=0 \quad\left(t \in \mathbb{R}_{+}\right)
$$

where $q, r$ are as for $(1)$ and $f(u) u>0$ for $u \neq 0$ such that

$$
f(u) \geq k u^{\lambda} \quad \text { for } u \in \mathbb{R}_{+}
$$

for some $\lambda \geq 1$ and $k>0$. The prototype of such an extension is the function $f(u)=e^{u}-1$ for $u \geq 0$.

Theorems 1-4 read for (1') as follows.

Theorem $1^{\prime}$ Let $\lambda \geq 1, r(t)<0$ and (11) hold for $t \in \mathbb{R}_{+}$. Assume that either (i) (12), or (ii) (13) and (14) hold. Then problem (1'), (3), (4) has a solution for any $c>0$.

Proof of Theorem $1^{\prime}$ It is analogous to the proofs of Proposition 1 and Theorem 1 replacing the nonlinearity $\left|y_{1}(t)\right|^{\lambda}(t) \operatorname{sgn} y_{1}(t)$ in system (10) by $f\left(y_{1}(t)\right)$. Lemma 1 remains to hold as a sufficient condition for (3).

Theorem $2^{\prime}$ Theorem 2 remains to hold for $\left(1^{\prime}\right)$ without assuming (50).

Proof of Theorem 2' In the proof of claim (a) of Theorem 2, we consider system (20) where the nonlinearity $\left|y_{1}(t)\right|^{\lambda}(t) \operatorname{sgn} y_{1}(t)$ is replaced by $f\left(y_{1}(t)\right)$. The proof of claim (b) of Theorem 2 is the same for the nonlinearity $f$.

Theorem $3^{\prime}$ Theorem 3 remains to hold for $\left(1^{\prime}\right)$.

Proof of Theorem $3^{\prime}$ Let $x$ be a positive solution of $\left(1^{\prime}\right)$ on $[a, \infty)$. Then $v=x$ is a solution of the equation

$$
v^{(4)}(t)+q(t) v^{(2)}(t)+R(t) v^{\lambda}(t)=0,
$$

where

$$
R(t)=\frac{r(t) f(x(t))}{x^{\lambda}(t)} \geq k r(t) \quad \text { for } t \geq a .
$$

Now we apply Theorem 3 to (51). 
Theorem 4' Let the assumptions of Theorem 4 hold. Then (1') has no eventually positive solutions.

Proof of Theorem 4' It is similar to the one of Theorem 4. In view of (52), the estimate (38) holds and the energy function $F$ is the same.

\section{Competing interests}

The authors declare that they have no competing interests.

\section{Authors' contributions}

Both authors contributed equally to the manuscript and read and approved the final draft.

\section{Acknowledgements}

Supported by the grant GAP 201/11/0768 of the Czech Grant Agency.

Received: 13 November 2012 Accepted: 16 March 2013 Published: 12 April 2013

\section{References}

1. Kiguradze, I, Chanturia, TA: Asymptotic Properties of Solutions of Nonautonomous Ordinary Differential Equations. Kluwer Academic, Dordrecht (1993)

2. Mawhin, J, Zanolin, F: A continuation approach to fourth order superlinear periodic boundary value problems. Topol. Methods Nonlinear Anal. 2(1), 55-74 (1993)

3. Gupta, C, Mawhin, J: Weighted eigenvalue, eigenfunctions and boundary value problems for fourth order ordinary differential equations. In: Recent Trends in Differential Equations. World Sci. Ser. Appl. Anal., vol. 1, pp. 253-267. World Scientific, River Edge (1992)

4. Ahlbrandt, CD, Hinton, DB, Lewis, R: The effect of variable change on oscillation and disconjugacy criteria with applications to spectral theory and asymptotic theory. J. Math. Anal. Appl. 81, 234-277 (1981)

5. Agarwal, RP, O'Regan, D: Infinite Interval Problems for Differential, Difference and Integral Equations. Kluwer Academic, Dordrecht (2001)

6. Elias, U: Oscillation Theory of Two-Term Differential Equations, vol. 396. Kluwer Academic, Dordrecht (1997)

7. Kiguradze, I: An oscillation criterion for a class of ordinary differential equations. Differ. Uravn. (Minsk) 28, 201-214 (1992)

8. Bartušek, M, Cecchi, M, Došlá, Z, Marini, M: Asymptotics for higher order differential equations with a middle term J. Math. Anal. Appl. 388, 1130-1140 (2012). doi:10.1016/j.jmaa.2011.10.059

9. Bartušek, M, Cecchi, M, Došlá, Z, Marini, M: Fourth-order differential equation with deviating argument. Abstr. Appl. Anal. 2012, Article ID 185242 (2012)

10. Berchio, E, Ferrero, A, Gazzola, F, Karageorgis, P: Qualitative behavior of global solutions to some nonlinear fourth order differential equations. J. Differ. Equ. 251, 2696-2727 (2011)

11. Hartman, P: Ordinary Differential Equations. Wiley, New York (1964)

12. Chanturia, TA: On monotone solutions of a system of nonlinear differential equations. Ann. Pol. Math. 37, 59-70 (1980) (in Russian)

13. Kusano, T, Naito, M, Wu, F: On the oscillation of solutions of 4-dimensional Emden-Fowler differential systems. Adv. Math. Sci. Appl. 11(2), 685-719 (2001)

14. Naito, M, Wu, F: A note on the existence and asymptotic behavior of nonoscillatory solutions of fourth order quasilinear differential equations. Acta Math. Hung. 102, 177-202 (2004)

15. Astashova, IV: Uniform estimates for positive solutions of quasi-linear ordinary differential equations. Izv. Ross. Akad. Nauk, Ser. Mat. 72(6), 85-104 (2008) (Russian); translation in Izv. Math. 72(6), 1141-1160 (2008)

16. Chanturia, TA: On singular solutions of nonlinear systems of ordinary differential equations. Colloq. Math. Soc. János Bolyai 15, 107-119 (1976)

17. Bartušek, M, Došlá, Z: Remark on Kneser problem. Appl. Anal. 56(3-4), 327-333 (1995)

18. Borůvka, O: Linear Differential Transformationen 2. Ordung. VEB, Berlin (1967)

doi:10.1186/1687-2770-2013-89

Cite this article as: Bartušek and Došlá: Asymptotic problems for fourth-order nonlinear differential equations.

Boundary Value Problems 2013 2013:89. 